\title{
La compleja tarea de evaluar la oralidad: una propuesta de evaluación auto/formativa
}

\author{
The complexity of orality evaluation: a design of self-training assessment
}

Yolima Gutiérrez Ríos *

Recibido: 10 de febrero de 2013 / Aceptado: 15 de marzo de 2013

\begin{abstract}
Resumen
La evaluación de la oralidad en los diferentes ciclos escolares es una tarea compleja y poco frecuente debido al predominio de una tradición escolar que poco ha avanzado en la enseñanza y aprendizaje de las destrezas comunicativas de hablar y escuchar en contextos formales de uso. En Colombia, en los últimos años, ha surgido un creciente interés por parte de docentes y colectivos de investigación hacia el desarrollo de propuestas de intervención orientadas a comprender la complejidad del qué, cómo, para qué y el porqué de la oralidad como actividad discursiva fundamental en la educación lingüística. En este sentido, el presente artículo plantea dos dispositivos, uno relacionado con la autoformación docente en investigación individualizada y el otro que tiene que ver con el desarrollo de proyectos de evaluación formativa, como estrategias para favorecer desarrollar y evaluar la competencia discursiva oral de niños y jóvenes.
\end{abstract}

Palabras clave: oralidad, concepciones, didáctica, evaluación y reflexión.

\section{Abstract}

The assessment of orality in different school levels is a complex task and not often approached, due to a predominant school tradition that has moved forward only a few steps in terms of teaching-learning communicative skills such as speaking and listening in formal contexts of usage. During the past few years in Colombia, a growing interest on developing classroom intervention proposals has emerged among teachers and research groups. These proposals aim to understand the complexity of orality as a fundamental discursive activity in linguistic education from the inquiries 'what', 'how', 'what for' and 'why'. In that regard, the following article raise two dispositives, the first one related to teachers self-formation on individual research; the second one, involves formative assessment projects development as strategies to encourage building up and assessing the oral discursive skill in children and young people.

Key words: orality, conceptions, teaching, assessment and reflection

\section{La reflexión sobre las concepciones en torno a la enseñanza de la oralidad}

Partimos de reconocer que en la escuela colombiana actual, la oralidad no es abordada como objeto de enseñanza permanente y por tanto, como un aprendizaje progresivo e intensivo, dado que se trata de un dominio discursivo con una débil tradición educativa derivada de circunstancias histórico-culturales y pedagógicas descritas en una investigación anterior (Gutiérrez, 2011). Sin embargo, esta ausencia de prácticas pedagógicas enfocadas al desarrollo de la lengua oral formal ha convocado en los últimos años a maestros

\footnotetext{
* Profesora de la Universidad de La Salle, Doctora en Educación. Universidad Distrital Francisco José de Caldas. Correo electrónico: yolimagr@ yahoo.es.
} 
investigadores en la ejecución de propuestas pedagógicas y didácticas orientadas a comprender los modos de apropiación de esta manifestación del lenguaje. Dicho avance nos ha permitido constatar la dificultad para objetivar los procesos de comprensión y producción oral y en consecuencia para evaluarlos, esto indica que estamos frente a una compleja tarea, para la que no todos los docentes nos sentimos preparados.

Por ello, urge revisar los planes de formación y actualización del profesorado, especialmente de los docentes de lengua materna, de preescolar y primaria, desde la perspectiva de acercarlos al estudio la reflexión sobre las repercusiones de la enseñanza de la lengua oral, tanto en el desarrollo personal, escolar y social de los estudiantes, como en los mismos procesos de adquisición y desarrollo de la lengua escrita ${ }^{1}$. Se trata de construir un "conocimiento didáctico del contenido lingüístico-discursivo oral" (Gutiérrez, 2011), representado en la capacidad para apropiar la naturaleza interdisciplinar de la oralidad e integrar sus dimensiones, retórica y lingüístico-discursiva; antropológica y sociocultural y por supuesto, literaria, a las características del contexto escolar y social particular.

Consideramos que es necesario que los programas de formación docente, a nivel de pregrados y posgrados, tengan en cuenta los sistemas de concepciones y saberes de los profesores sobre la ciencia, la enseñanza y el aprendizaje, dadas sus implicaciones en la construcción del conocimiento escolar. Específicamente, es primordial la detección, la potenciación y la movilización de concepciones ${ }^{3}$ en torno a la actividad oral de la escuela, lo cual contribuiría en gran medida a la construcción de una didáctica de la oralidad desde la misma experiencia investigativa de los maestros.

Por ejemplo, en la caracterización y el análisis de las concepciones didácticas y disciplinares de profeso- res de diferentes áreas y ciclos escolares en torno a la oralidad, hemos encontrado una naturalización de sus usos debido a que, por una parte, se concibe como una práctica cotidiana y connatural a los actores escolares $y$, por ende, se asume su potenciación desde su función mediadora en los procesos de enseñanza y aprendizaje. Por otro lado se advierte que se incorporan de manera espontánea, prácticas relacionadas con lecturas en voz alta, juegos de palabras, conversaciones formales, asambleas, exposiciones orales, debates, entre otras, sin contar con mayores estrategias para su articulación y progresión con miras a cualificar la adquisición y desarrollo oral.

Este carácter instrumental dado a la oralidad la aleja, en primer lugar, de la posibilidad de comprender su naturaleza discursiva y de la oportunidad de incidir en las formas de interactuar en diversos contextos de uso y, en consecuencia, del desarrollo de una capacidad reflexiva del educando sobre su propia práctica discursiva.

Por todo lo anterior es necesario pensar en la oralidad como objeto de enseñanza y aprendizaje, sin que ello signifique la pérdida de su espontaneidad y autenticidad; por el contrario, reconocer el carácter fugaz y cambiante del discurso oral impone un desafío para aquel que pretenda aportar a la mejora de la capacidad comunicativa de los estudiantes. Además, es necesario imponernos a cierto escepticismo que ronda en la comunidad académica respecto a la importancia de una didáctica de la oralidad como campo de formación e investigación y de bases teórico-prácticas sólidas para la enseñanza de la lengua oral en la educación básica, media y superior, lo cual confirma la necesidad de estudiar el conocimiento profesional del profesor de lengua y particularmente, su conocimiento didáctico del contenido lingüístico-discursivo oral.

1 Cabe señalar que algunas universidades, como la Pontificia Universidad Javeriana, han empezado a incorporar en los currículos de formación docente cátedras relacionadas con la didáctica de la oralidad.

2 Entendemos las concepciones como el conjunto de ideas y formas de actuar de los profesores en torno a la enseñanza y aprendizaje de una materia o de un tema específico de esta. 
Ahora bien, si nos situamos en el desarrollo de experiencias espontáneas o planeadas sobre la actividad oral, surge de inmediato la pregunta por criterios de evaluación que determinen los comportamientos lingüísticos subyacentes. Esta simultaneidad evidencia que la creación de situaciones comunicativas orales, bien sea reales o simuladas, es en sí misma, una evaluación de la comprensión y la producción oral; es decir, enseñar y aprender lengua oral implica, tanto para los estudiantes como para el maestro, iniciar un proceso de evaluación reflexiva y sistemática de discursos orales (propios y aludidos) y actuaciones. En este punto, cobra gran importancia, la reflexión del maestro sobre su discurso y su práctica pedagógica.

En la observación y la escucha de prácticas discursivas orales espontáneas o planeadas subyace la actividad cognitiva (reflexión sobre el papel de las personas, los discursos y los contextos) y la actividad metacognitiva (reflexión sobre las estrategias discursivas orales). Se trata de dos actividades cuya reflexión deliberada posibilita analizar el desarrollo de la competencia discursiva oral de los estudiantes. La reflexión sobre qué evaluar, cómo y cuándo hacerlo, puede ayudarle al docente a establecer cuáles son las competencias orales que se deben cualificar. Esta labor investigativa remite a la necesidad de situar el problema de la enseñanza y la evaluación de la oralidad como objetos de conocimiento y de reflexión desde la perspectiva de recontextualizar los avances logrados por las Ciencias del Lenguaje en cuanto al análisis de la lengua y el discurso oral para ponerlos al servicio de la educación lingüística.

\section{La evaluación auto formativa docente en investigación}

En concordancia con el planteamiento anterior, consideramos que la formación y la actualización docente deben apuntar a la investigación ${ }^{3}$ de problemas profesionales abiertos y complejos. Se trata de una investigación formativa que en torno a la enseñanza y la evaluación de la oralidad desde la perspectiva de fortalecer la construcción del "conocimiento didáctico del contenido lingüístico-discursivo oral", pueda a su vez constituir una línea de investigación potente, dado el marco de reflexión-acción-reflexión del que emerge.

La siguiente propuesta de autoformación docente en didáctica de la lengua oral surge de dos investigaciones realizadas en consideración de las concepciones y las prácticas de la oralidad de docentes de Lengua Castellana en los ciclos tres, cuatro y cinco de diez instituciones de educación pública de Bogotá ${ }^{4}$ Se realizaron entrevistas y observaciones de clase, cuyo análisis de contenido conllevó a diversas conclusiones, a partir de las cuales surge un dispositivo de autoformación docente, dicha realización se concreta en tres estrategias y modalidades de autoformación docente en investigación cifradas en los principios de autonomía y desarrollo profesional autodirigido, tales como: la autoformación docente en investigación individualizada, la autoformación docente en investigación grupal y la autoformación docente en investigación en red. La autoformación se entiende como un proceso de autoaprendizaje determinado por el ritmo individual, el cual se articula a unos objetivos colectivos. La concreción

\footnotetext{
3 Se plantea una investigación educativa en la que el profesorado toma parte activa como sujeto que interpreta los fenómenos educativos y no como un objeto de estudio. Cabe agregar la precisión que hace Stenhouse (1984) entre investigación en educación, aquella que se realiza en el marco de un proyecto educativo con unos sujetos que participan en él e investigación sobre la educación, aquella que se realiza para aportar al conocimiento de la educación

4 "Análisis de las concepciones disciplinares y didácticas sobre la lengua oral en la escuela colombiana actual: un estudio sobre el desarrollo de la competencia discursiva oral en la Educación Media" y "Evaluar lo oral para cualificar las prácticas de enseñanza aprendizaje de la lengua materna”, adelantadas por miembros del Grupo de investigación Lenguaje, Cultura e Identidad, de la Universidad Distrital Francisco José de Caldas..
} 
de este dispositivo implica la realización de principios éticos y didácticos orientados a consolidar una autonomía profesional en el profesor y la evolución del perfil de formador de formadores al rol de acompañante. A continuación, profundizaremos en la autoformación docente en investigación individualizada.

\section{La autoformación docente en investigación individualizada}

Este tipo de formación docente corresponde a un ciclo inicial propio del autoaprendizaje del profesor en ejercicio y a un proceso de indagación personal y presencial, organizado en textos, cursos o talleres, que le proporcionen instrumentos metodológicos para analizar su saber experiencial y para construir estrategias que le posibiliten solventar sus problemas. El carácter individualizado del trabajo implica un seguimiento comprometido con unos niveles y ritmos propios y con el tratamiento de problemas curriculares relacionados con la oralidad.

Se entiende como un ciclo inicial aquel que requiere el acompañamiento de un profesor o asesor que oriente la reflexión en la acción en los dos niveles propuestos por Camps, Ríos y Cambra (2004) con algunas modificaciones. Teniendo como preámbulo la caracterización y el análisis de las concepciones que emergieron de esta experiencia, el primer nivel parte del análisis de una experiencia de aula representada como juego de saber donde se identifiquen las reacciones inmediatas del profesor ante devoluciones de los estudiantes o situaciones de ocurrencia en el aula alrededor de los aprendizajes de la lengua oral. El segundo nivel consiste en el análisis de las maneras como el profesor redefine el juego didáctico o lo reorganiza en la marcha ante las eventualidades que se le presentan. Esta reflexión en la acción conlleva a identificar y analizar las concepciones del profesor sobre la enseñanza y el aprendizaje de la lengua oral.

Esta modalidad formativa opera también como estrategia de acercamiento a aquellos profesores en ejercicio que por diversas circunstancias no se vinculan a los planes y programas de actualización docente que ofrecen las instituciones de formación docente o los institutos o grupos de investigación. A pesar de la corta duración de un curso, seminario, taller o conferencia, son espacios en los que se comparten experiencias profesionales, se crean inquietudes, se combinan momentos para el diseño de materiales didácticos con otros de aplicación y de reflexión crítica, que logran el cuestionamiento de los profesores sobre algunos componentes de su actividad pedagógica y los incentivan a realizar acciones de cambio (Porlán y Rivero, 1998).

Se parte de la selección de una situación problémica derivada de la realidad del aula, la cual se precisa mediante las preguntas sobre ¿qué sabemos los profesores acerca de la enseñanza y el aprendizaje de la lengua oral en la educación inicial? y ¿qué lengua oral enseñamos y cómo? Se considera que estos interrogantes y los que puedan derivarse de ellos posibilitan centrar la mirada en el peso que tienen las ideas, las reglas de acción y las actitudes de los docentes en su desempeño profesional.

En suma, con este ciclo de formación se pretende una toma de conciencia por parte de los profesores frente a la importancia de caracterizar y reestructurar su sistema de concepciones e hipótesis curriculares, tal como se ve en la tabla 1.

De este modo, la construcción del "conocimiento didáctico del contenido lingüístico-discursivo oral" de carácter reflexivo y sistemático adquiere relevancia mediante la investigación, la cual puede desembocar en una actitud reflexiva del profesor o en una actividad investigativa permanente. Es este el sentido propuesto por Imbernon (2009), quien aboga por una formación como desarrollo profesional que oriente al profesorado hacia la resignificación de conocimientos, destrezas y actitudes para lograr formar profesionales reflexivos o investigadores de su propia práctica pedagógica, a fin de comprender la enseñanza y la realidad social de forma comunitaria.

Esta propuesta de actuación en el aula frente a la enseñanza de la oralidad, contempla la complejización de las actividades de hablar y escuchar, favoreciendo la interacción oral, el intercambio de saberes socioculturales y lingüísticos, el derecho a ser reconocido por el 
otro, a ser escuchado, a que se valore su registro lingüístico y a conocer otras alternativas o formatos de habla y su adecuación a los diversos contextos y en últimas, al dominio de los distintos géneros discursivos orales. ${ }^{5}$

Tabla 1. Ciclo inicial o ciclo de autoformación docente en investigación individualizada

\begin{tabular}{|c|}
\hline OBJETIVO GENERAL \\
\hline $\begin{array}{l}\text { Caracterizar diferentes situaciones de enseñanza y aprendizaje de la lengua oral, con el fin de suscitar } \\
\text { diversos cuestionamientos alrededor de determinadas concepciones disciplinares y didácticas de la ora- } \\
\text { lidad que pueden constituir barreras o ayudas para interpretar la realidad del aula. }\end{array}$ \\
\hline Objetivos específicos \\
\hline \multirow{2}{*}{$\begin{array}{l}\text { Primer nivel. Analizar situaciones de ocurrencia en el aula relacionadas con la comprensión y producción } \\
\text { oral de los estudiantes, con el fin de identificar reacciones inmediatas del profesor y modos de gestionar } \\
\text { los aprendizajes. } \\
\text { Segundo nivel. Caracterizar las maneras como cada profesor afronta las potencialidades y dificultades } \\
\text { relacionadas con la interacción oral de los estudiantes: el habla y la escucha. }\end{array}$} \\
\hline \\
\hline Situación problémica \\
\hline $\begin{array}{l}\text { A menudo nos quejamos de la pobreza lingüística de nuestros estudiantes y lamentamos su dificultad } \\
\text { para construir discursos orales coherentes e incluso enunciados con cierto grado de claridad, pertinencia } \\
\text { y adecuación a la situación comunicativa en cuestión. Asimismo, nos preocupan aquellos estudiantes } \\
\text { que no logran comprender o no se interesan por los diferentes discursos orales que circulan en el aula } \\
\text { (lecturas realizadas en voz alta, explicaciones, exposiciones, participaciones, etc.), así como aquellos que } \\
\text { poco o nunca intervienen en clase (a partir de preguntas, objeciones, aclaraciones, explicaciones, etc.). }\end{array}$ \\
\hline Itinerario de problemas \\
\hline \multirow{2}{*}{$\begin{array}{c}\text { Nivel } 1 \\
\text { ¿Qué sabemos los profesores acerca de la enseñanza y el aprendizaje de la lengua oral? }\end{array}$} \\
\hline \\
\hline $\begin{array}{l}\text { ¿Es posible enseñar y aprender la lengua oral?; ¿a qué obedece el interés actual y súbito por lo oral?, } \\
\text { ¿la enseñanza de lo oral es una tarea nueva o renovada?; ¿es necesario potenciar los modos de hablar y } \\
\text { escuchar de los estudiantes?; ¿sobre cuáles dispositivos teóricos y metodológicos apoyarse para tomar } \\
\text { decisiones durante una sesión de clase centrada en la observación, descripción y/o análisis del discurso } \\
\text { oral de los estudiantes?, ¿en qué documentos, libros de texto o materiales curriculares apoyarse para } \\
\text { orientar el desarrollo de la oralidad? }\end{array}$ \\
\hline
\end{tabular}

(Continúa)

\footnotetext{
5 Estos se caracterizan, según Castellá y Vilá (2005), por presentar enunciados que tienen cierta similitud en su contenido, estilo y composición verbal, y se clasifican como géneros discursivos primarios (interacciones orales espontáneas e informales), géneros discursivos secundarios (interacciones orales reflexivas y formales) y géneros discursivos intermedios (comparten en proporciones variadas características de los géneros discursivos primarios y secundarios).
} 
Nivel 2

¿Qué de lengua oral enseñamos y cómo?

Contenidos: ¿cuáles son los obstáculos que surgen en la programación de contenidos de conocimiento de la lengua oral?; ¿qué lugar ocupa la oralidad en el currículo institucional y en los documentos de política curricular, en libros de texto y/o en los materiales curriculares?

Finalidades: ¿por qué enseñar y aprender lengua oral?

Tiempo didáctico: ¿qué tiempo se le debe concebir a lo oral?

Condiciones para la enseñanza de la lengua oral: ¿qué condiciones se requieren para introducir en las aulas una enseñanza explicita, sistemática y evaluable de la lengua oral?

Actividades: ¿cuáles actividades pueden favorecer el desarrollo de la escucha, el habla y la interacción oral de los estudiantes?, ¿aunque en la mayoría de las clases, se debate, se pregunta y se opina, ¿por qué los avances en la comprensión y producción oral de los estudiantes son mínimos?

Estrategias didácticas: ¿lo oral se prepara, cómo?; ¿qué estrategias diseñar para hacer progresar el discurso oral de los estudiantes sin que se pierda la "naturalidad" de la comunicación en clase?; ¿cómo lograr la participación y la cooperación de todos los estudiantes y la progresión de cada uno?

Evaluación: ¿cómo evaluar la oralidad de los estudiantes?, ¿cómo hacer el balance de las potencialidades y las necesidades de los estudiantes respecto a su comprensión y producción oral?

Fuente: elaboración propia.

Se trata de una progresión de la enseñanza y aprendizaje de la lengua oral, apoyada en tres perspectivas metadisciplinares (constructivista, sistémica y compleja y la perspectiva crítica) que fundamentan el "modelo didáctico de investigación en la escuela" (Porlán y Rivero, 1998), las cuales se concretan en las dos actitudes mentales que propone Shön (1983) como vía para que el profesor construya un nuevo conocimiento: "la reflexión en la acción y la reflexión sobre la acción didáctica".

\section{La evaluación formativa y auténtica de la oralidad}

Compartimos con María Elena Rodríguez (1995) que el niño llega al contexto escolar provisto de una competencia lingüística que le permite, salvo en el caso de graves patologías, comprender y producir distintos enunciados; interactuar con relativo éxito en distintos contextos de comunicación y aprender de forma espontánea algunos de los usos habituales que rigen la lengua oral de su entorno familiar y social. Se trata entonces, de saberes lingüísticos, textuales y pragmáticos con una menor o mayor elaboración, dependiendo del entorno familiar y sociocultural de cada pequeño, hecho que compromete a los profesores de la primera infancia en la tarea inicial de caracterizar esta desigualdad comunicativa.

La superación de dicha desigualdad oral de los niños y niñas que ingresan a la escuela supone una perspectiva política de la oralidad y de la evaluación, esta última constituye un punto de partida fundamental para pasar de un aprendizaje espontáneo, a una adquisición y desarrollo de la lengua oral formal. Indiscutiblemente, esta pretensión configura la necesidad de prácticas innovadoras, cuyo éxito está determinado en gran medida, por las concepciones de enseñanza y aprendizaje de la lengua oral de quienes están comprometidos con la formación de las presentes y futuras generaciones. 
Sin duda, evaluar la oralidad es una de las tareas más difíciles, dado su carácter subjetivo; se requiere entonces una evaluación objetiva del estado inicial de la competencia discursiva oral de los pequeños que considere la creación de situaciones comunicativas orales orientadas a identificar sus potencialidades, dificultades y expectativas, a fin de diseñar, desarrollar y evaluar propuestas de intervención efectivas. Acercarnos a una objetivación de la subjetividad de la evaluación de lo oral exige herramientas fiables (tablas de especificaciones, lista de cotejo, rúbricas o matrices de valoración, etc.) y dispositivos tecnológicos (grabadora, videocámara, etc.) que rescaten la autenticidad y la evanescencia de la oralidad.

Al plantear el carácter objetivo de la evaluación de lo oral, nos situamos en una evaluación formativa que contempla la interrelación entre las actividades inherentes a las situaciones didácticas diseñadas por el maestro y los progresivos avances que logra el estudiante en su proceso de aprendizaje. $\mathrm{Al}$ respecto, Antonio Mendoza (1998), plantea:

La actividad formativa de la evaluación motiva que interaccionen las aportaciones del docente y la actividad del aprendiz, de modo que se ponga de manifiesto la eficacia de la actividad de ambos; por ello, los efectos de esta modalidad tienen repercusión inmediata en la construcción y regulación de aprendizajes (p. 13).

Esta perspectiva formativa de la evaluación de la oralidad, como cabe suponer la plantea al estudiante una evaluación significativa de su aprendizaje y al maestro una participación decidida y renovada de sus prácticas pedagógicas. La pretensión de una postura crítica del profesor sobre su propia práctica permite integrar sus reflexiones al proceso de evaluación en este caso de la oralidad, lo cual implica:

- Una reflexión crítica en cuanto al tratamiento actual de la evaluación de la lengua oral en el aula.

- Un conjunto de estrategias y procedimientos (criterios e indicadores) para orientar la eva- luación del desempeño comunicativo de los participantes en contextos auténticos de enseñanza y aprendizaje.

La categoría de evaluación de la oralidad reconoce diversas situaciones de enunciación oral con rasgos comunes. De acuerdo con Rodríguez (1995), los principales son:

- Los interlocutores construyen una relación interpersonal: la copresencia o participación simultánea de los participantes es determinada por sus características psicosociales y culturales (roles, estatus, hábitos...)

- El contexto compartido: se comparte un aquí y un ahora (espacio y tiempo) que puede existir o existe realmente.

- Las intenciones comunicativas: los interlocutores negocian, construyen y/o deconstruyen y su desempeño lingüístico se ve enriquecido por la capacidad de retroalimentación constante de hablar y escuchar.

En consecuencia, la evaluación de lo oral exige procesos de realización auténticos, diversos y variados, según la capacidad de comprensión y producción oral de los interlocutores. La evaluación de lo oral tiene un carácter multirreferenciado y complejo; se trata entonces de pensar la evaluación no desde la idea de medir o emitir un juicio sobre un saber, a partir de un instrumento específico, sino de acudir a una estrategia que posibilite crear unas condiciones para llevar a cabo un proceso de reflexión en torno al papel que desempeña la oralidad en la cotidianidad del aula desde la experiencia particular de los maestros (sus modos de pensar y actuar), es decir, desde sus concepciones sobre la enseñanza y el aprendizaje de la lengua oral en los primeros grados de escolaridad.

Sin duda alguna, estamos frente a la evaluación como práctica cultural que pone en diálogo saberes y experiencias a la luz de elementos epistemológicos en 
función de facilitar interpretaciones críticas, diseñar situaciones problémicas y en últimas buscar un acercamiento a la autoformación docente en didáctica de la lengua oral. Si la evaluación es concebida desde la problematización de las actividades de enseñanza y aprendizaje y en este caso sobre aspectos relacionados con la lengua oral estamos frente a una estrategia y una vía para enriquecer la práctica pedagógica.

Para lograr un espectro amplio de la evaluación de la oralidad, Atorresi y Ravela (2009) proponen los proyectos de evaluación formativa como una alternativa para la planeación de la enseñanza y la promoción de aprendizajes significativos y duraderos, mediante "una secuencia de unidades puntuales o breves de enseñanza, seguidas por momentos también puntuales de evaluación del aprendizaje". Esta alternativa, posibilita interconectar los procesos de enseñanza, aprendizaje y evaluación, y, asimismo, captura un amplio espectro de desempeños y se adecúa a los propósitos de formación, a las características de los estudiantes y al contexto escolar particular.

Los proyectos de evaluación formativa son auténticos, dado que resuelven situaciones problema, muy semejantes a la vida extraescolar, es decir ocurre en situaciones de actuación real. Para tal fin se organiza en fases, de modo que los ajustes y los procesos de evaluación y devolución requieran una intervención mutua que incluye acciones autoreflexivas.

\section{Conclusiones}

Lo expuesto anteriormente ratifica la importancia de enfocar la enseñanza y evaluación de la lengua oral desde una visión complementaria que interrelacione elementos lingüístico-discursivos, paralingüísticos, kinésicos, proxémicos y metacognitivos de los usos orales espontáneos y los usos orales formales de manera secuencial y progresiva en favor del desarrollo de la competencia discursiva oral de los niños, lo cual implica no solo asumir la oralidad como objeto de estudio, sino también como objeto de enseñanza.
Esto es, lograr la combinación adecuada y la transformación didáctica de un conocimiento disciplinar (visión complementaria que interrelacione elementos lingüístico-discursivos, paralingüísticos, kinésicos, proxémicos y metacognitivos de los usos orales espontáneos y los usos orales formales de manera secuencial y progresiva), un conocimiento didáctico (situaciones de enseñanza que privilegia, desarrolla y evalúa) y un conocimiento pedagógico (principios y estrategias generales de manejo y gestión de la clase).

Se espera que el desarrollo de experiencias pedagógicas y didácticas relacionadas con la oralidad no constituya una práctica esnobista, realizada por unos pocos, aquellos que cuentan con las condiciones para abordar tan compleja tarea, sino que poco a poco todo el profesorado, vaya incorporando de manera sistemática y progresiva el desarrollo de la competencia discursiva oral de los niños, niñas y jóvenes.

Cabe señalar que se trata de procesos que comportan elementos básicos para pensar una didáctica de la lengua oral, lo cual implica el diseño secuencial de actividades evaluativas que nos informen de las capacidades respecto a su comprensión y producción oral. Desde esta perspectiva, la evaluación mantiene la intención de mejorar los procesos de enseñanza y aprendizaje y, por ello, se considera formativa.

Así pues, la constante indagación cooperativa coadyuva en la generación de conocimiento pedagógico cada vez más complejo y riguroso, y sobre todo, consecuente con la problemática cotidiana del contexto escolar, lo cual significa un acercamiento a un conocimiento profesional deseable.

\section{Reconocimientos}

Este artículo presentan los resultados de la investigación Evaluar lo oral para cualificar las prácticas de enseñanza aprendizaje de la lengua materna, adelantada por el Grupo de investigación Lenguaje, Cultura e Identidad, de la Universidad Distrital Francisco José de Caldas. 


\section{Referencias}

Atorresi, A. y Ravela, P. (2009). Los proyectos de evaluación formativa y auténtica. Montevideo: Instituto de Investigaciones en Evaluación, Universidad Católica del Uruguay.

Camelo, M., Cárdenas, E., Torres, M., Gutiérrez, Y., Rodríguez, M. y Pinilla, R. (2011). Hallazgos iniciales sobre la evaluación de la lengua oral en el aula. Revista Enunciación, 16(1), 70-83.

Camps, A., Ríos, I. y Cambra, M. (2004). Recerca i formació en didáctica de la llengua. Lenguaje, 32, 7-27.

Castellá J. y Vilá, M. (2005). La lengua oral formal: características lingüísticas y discursivas. En M. Vilá C., J. Ballesteros, A. Castellá, A. Cros y M. Grau (Coord.), El discurso oral formal (pp. 25-36). España: Grao.

Gutiérrez, Y. (2011). Análisis de las concepciones disciplinares y didácticas sobre la lengua oral en la es- cuela colombiana actual: un estudio sobre el desarrollo de la competencia discursiva oral en la Educación Media (Tesis Doctoral). Universidad Distrital Francisco José de Caldas, Bogotá.

Imbernon, F. (2009). Mejorar la enseñanza y el aprendizaje en la universidad. Barcelona: Editorial Octaedro, Cuadernos de docencia universitaria 14.

Mendoza, A. (1998). Concepción y creencias de la evaluación en el docente. Revista Interuniversitaria de formación del profesorado, 33, 107-120.

Porlán, R., \& Rivero, A. (1998). El conocimiento de los profesores. Sevilla: Díada.

Rodríguez, M. (1995). "Hablar” en la escuela: ¿Para qué?... ¿Cómo? Lectura y Vida. Revista Latinoamericana de Lectura, 16 (3), 1-11.

Schön, D. (1983). The Reflective Practitioner: How Professionals Think in Action. NY: Basic Books.

Stenhouse, L. (1984). Investigación y desarrollo del currículo. Madrid: Morata. 